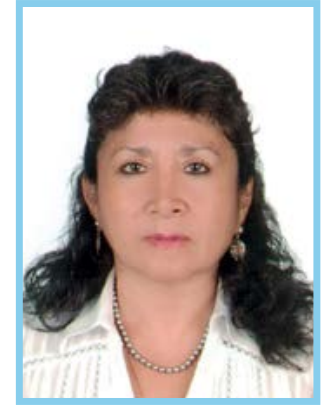

Dra. Olga González

Sarmiento

\section{LA EDUCACIÓN TRANSFORMA}

Si todos los niños tuvieran el mismo acceso a la enseñanza, el ingreso per cápita aumentaría un 23\% en los próximos 40 años (UNESCO).

\section{RESUMEN}

El presente artículo examina el aporte de la educación en la vida de las personas, en especial de las mujeres, citando interesantes datos estadísticos que plasman con claridad la diferencia existente en la vida de alguien que ha sido educado $y$ en quien no pudo acceder a ese beneficio.

La premisa que maneja la autora es que la educación transforma y transforma para bien, pero también advierte, que no se va a dar tal transformación si la educación que niños, jóvenes y/o adultos reciben no cuenta con niveles mínimos de calidad.

\section{ABSTRACT}

The present article studies the contribution of education in the lives of people, especially women, citing interesting statistics that reflect clearly the difference in the life of someone who has been educated and who could not access that benefit.

The premise that the author manages is that the education transforms and transforms for the better, but also warns that it will not provide such transformation if education to children, youth and/or adults receiving lacks with the minimum quality.

\section{PALABRAS CLAVE}

Educación, transformación, mujer.

\section{KEYWORDS}

Education, transformation, woman.

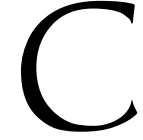
uando se habla de educación muchas veces los lugares comunes se refieren a una determinada serie de requerimientos: mayores sueldos para los maestros, mejor uso de la tecnología, utilización de metodologías más modernas y funcionales, número de alumnos que se matricularon, número de alumnos que desertaron, cantidad insuficiente del PBI destinado al sector, problemas con la calidad y cantidad de infraestructura disponible y un largo etcétera adicional.

Sin embargo, y desde mi experiencia, muchas veces dejamos de lado lo más importante y espero, imperecedero de la educación: el hecho de que transforma vidas.

Algunas personas con una ceja alzada quizás se pregunten: itransforma vidas, tanto así?, itanta influencia puede tener la educación?..., pruebas al canto...

Según datos de la UNICEF, educar a las niñas puede salvar millones de vidas, iy no es lo mismo que nos refiramos a los niños? Los datos recogidos por la UNICEF afirman que "entre 1990 y 2009 se salvó la vida de 2,1 millones de niños menores de cinco años, gracias a las mejoras introducidas en la educación de las niñas", ¿por qué razón?, pues ya se lo imaginará usted, si educamos a las futuras madres y ellas tienen conocimiento 
de determinadas enfermedades, serán capaces de identificarlas en sus inicios o, en el mejor de los casos, prevenirlas.

Y la diferencia la determinan no unos estudios a nivel superior, no, basta solo que la niña haya cursado estudios de educación primaria. "Las madres que han finalizado la enseñanza primaria tienen un $12 \%$ más de probabilidades que aquellas que no han recibido educación de buscar atención sanitaria apropiada cuando un hijo presenta síntomas de diarrea".

Según la UNICEF, existen países donde el aumento de un año de la educación materna se asocia con una disminución del $23 \%$ del número de menores de cinco años que mueren de neumonía.

Pero no se trata solamente de lo referido a la prevención de enfermedades, no es así, también el que una niña acceda a la educación la prepara para combatir al peligro que arrebata la tercera parte de las vidas infantiles a nivel mundial: la malnutrición.

Una madre perteneciente a los estratos más pobres de la población, con un presupuesto exiguo pero que siquiera ha asistido a la escuela para su educación primaria va a saber escoger aquellos alimentos que sean de bajo costo pero con mayor potencial nutricional. Una madre no educada quizás se vea obligada a pedir limosna en la calle y compre para ella y su bebé una bebida gaseosa, como muchas veces hemos podido ver en las calles, en vez de optar por otras alternativas más saludables, sobretodo, para su criatura.

Y, por supuesto, junto con el tema de la nutrición se encuentra íntimamente relacionado el tema de una buena higiene para la prevención de enfermedades. Algunos médicos, hoy en día, señalan que el simple hecho de lavarse las manos es condición para prevenir al menos un 50\% de las enfermedades asociadas a temas de contagio.
Por otro lado, y sin remontarnos al África Subsahariana o al Asia Meridional, aquí, mucho más cerca de nuestro país, en Honduras, se ha recogido datos acerca de niños con retraso en su crecimiento.

Tales datos mencionan que "las posibilidades de que un niño padezca retraso del crecimiento -estatura insuficiente para su edad- son del $54 \%$ si el nivel de educación de la madre es inferior al primario, mientras que descienden al $33 \%$ en caso de que la madre haya cursado la enseñanza primaria, y al 10\% cuando la madre tiene al menos educación secundaria.

Una madre que ha asistido a la escuela conocerá de la importancia de la lactancia materna el mayor tiempo posible, la posibilidad de obtener proteínas de los cereales, la necesidad de consumir alimentos propios de la región; por lo tanto, más económicos pero, igualmente alimenticios, como son, en nuestro caso, la quinua, la kiwicha, la kañihua.

Existen datos interesantes recogidos esta vez de Vietnam donde se afirma que "a la edad de un año -cuando los efectos adversos de la malnutrición en las perspectivas de futuro es probable que sean irreversibles-, los niños cuyas madres han alcanzado el primer ciclo de la enseñanza secundaria tienen menos de un 67\% de probabilidades de sufrir un retraso del crecimiento que aquellos cuyas madres no han recibido educación alguna”.

Sin embargo, el poder transformador de la educación no se queda únicamente en aspectos como la prevención del retraso en el crecimiento debido a una malnutrición o la prevención de una serie de enfermedades; indudablemente, no es así.

Quizás uno de los aspectos más importantes radica en que la educación dignifica a la persona a través del trabajo, es decir, le ayuda a conseguir mejores ofertas laborales y por tanto, ayuda a que 
se rompa el circuito cruel de la pobreza, que según los entendidos, sin ayuda de la educación, puede durar siete generaciones en la vida de una familia!

Es por este motivo que las personas que pertenecen a los sectores más desfavorecidos dentro de una sociedad son los que deberían de recibir la educación de mejor calidad, debido a que para ellos, la educación que reciban significa la posibilidad de pasar "de condiciones menos humanas a condiciones más humanas"; por tal motivo, ningún recorte de presupuesto debería mermar la calidad de educación que el Estado brinda a los sectores más desfavorecidos de la población.

$\mathrm{Al}$ respecto, UNICEF menciona que, si los estudiantes provenientes de países con ingresos bajos, al término de su escolaridad poseyeran competencias básicas en lectura, 171 millones de personas podrían salir de la pobreza, lo que equivaldría a una reducción del 12\% en términos de pobreza mundial.

Por otro lado, "en El Salvador, solo el 5\% de los adultos trabajadores con un nivel de instrucción inferior al primario tienen un contrato de empleo, frente al $47 \%$ de aquellos que han cursado la enseñanza secundaria”.

Así como sucede en El Salvador, en otros países como el nuestro es imprescindible que se brinde una educación de calidad que asegure al estudiante de educación básica (la única obligatoria y quizás la única que estudie con gran esfuerzo de su familia), una educación tan buena que permita que los pequeños estudiantes egresen con, por lo menos, el nivel de competencias básicas en lectura, que le permitan acceder a condiciones laborales mínimas y que no se conviertan, a la larga, en analfabetos funcionales sufriendo, de hecho, una merma en su potencial.

Por otro lado, y aunque muchos desearíamos que no fuera así, todavía existe la discriminación contra la mujer, muchos casos se cuentan pero, se me viene en este momento un recuerdo de hace cinco años más o menos.

Era un poco más de las 6:00 a. m. y, junto con una vecina, corríamos bordeando un parque cercano a nuestras casas cuando se nos acercó a toda velocidad un carro que nos obligó a saltar al jardín para evitar que nos arrollara y el conductor a todo pulmón gritó un insulto y nos dijo que "nos fuéramos a cocinar, en vez de perder el tiempo".

Por alguna razón de los múltiples ejemplos que podría dar, este es el primero que recuerdo, pero hay muchos más que usted o yo, o cualquiera podríamos citar. La mujer utilizada como objeto sexual o generador de morbo colectivo en determinados programas televisión o en la red, la exclusión de la mujer manifestada en menores sueldos o salarios con respecto al varón, o la presencia mayoritaria de varones en los más altos cargos a nivel empresarial o gubernamental.

Sin embargo, esta exclusión o discriminación de género se da con mayor fuerza aún cuando la mujer no ha recibido la preparación necesaria tanto a nivel escolar como en el nivel técnico o universitario.

Una exalumna me dijo una vez "la educación empodera, sino, mírame a mí..." y lo dijo con fuerza, con orgullo, se trata de una mujer de mediana edad que se encuentra estudiando su carrera de Psicología, me dio mucho gusto escuchar de ella esas palabras, efectivamente, la educación da fuerza, da conciencia de lo que uno es capaz de realizar, da oportunidades, da una nueva percepción de la vida y de lo que sucede a lo largo de ella.

La educación empodera, la educación TRANSFORMA, así, sencillamente, sin un tinte feminista en la afirmación, las palabras dichas por el valor de lo que expresan, la educación da la posibilidad de ser una mejor persona, una mujer más completa, una mujer que se valora y que 
puede ayudar a que otros, su familia, sus amigos, su sociedad, sean mejores desde su humilde labor del día a día, en el contexto que sea.

Además, es más probable que las niñas y las jóvenes instruidas conozcan sus derechos y dispongan de la confianza necesaria para exigirlos.

Y no solo se trata de confianza en sí mismas, sino también de libertad para tomar decisiones que afecten positivamente su vida, salud, integridad, supervivencia, tanto para ellas mismas como para sus hijos, en aras de labrarse un futuro mejor.

Pero, si hablamos de decisiones que afectan la vida, ¿cuáles son algunas de las cifras relacionadas con educación en el Perú?

En el 2003, sólo se matricularon en secundaria 2 millones 300 mil alumnos, es decir la mitad de aquellos que accedieron a la primaria. El acceso a educación secundaria en el campo es aún muy deficiente, pues sólo el $50 \%$ de ellos logra acceder, versus al casi $80 \%$ en zonas urbanas.

Igualmente, el $42 \%$ de los adolescentes pobres no van a la escuela secundaria, una cifra preocupante si se le compara con el 13\% característico de otros grupos socioeconómicos.

Por otro lado, las cifras afirman que un 33\% de los adolescentes, entre los 12 y 18 años, realiza actividades económicas y la mitad de ellos combina el trabajo con el estudio.

Se estima que en el ámbito rural, el 40\% de niños y adolescentes en el Perú trabaja en actividades agropecuarias, con serios efectos en su escolaridad (49\% de los que trabajan y asisten a la escuela tienen uno o más años de atraso escolar).

Finalmente, el $62 \%$ de las adolescentes trabajadoras del hogar (cuyo número es difícil de estimar) no asiste a la escuela y tiene una jornada semanal de 57 horas.

Y zqué nos dicen las estimaciones sobre toma de decisiones en lo que se refiere a embarazos adolescentes?

En nuestro país, hay 162 mil madres adolescentes, según cifras del INEI. Sus edades fluctúan entre los 12 y 19 años, encontrándose que en el área rural el $20 \%$ de adolescentes en este rango ya son madres.

La maternidad adolescente es cuatro veces mayor en adolescentes sin educación (37\%) que con educación secundaria. Lo alarmante es que en el departamento de Loreto, los indicadores muestran que el $26 \%$ de las adolescentes ya son madres de familia.

En la sierra el porcentaje es de $15 \%$ y en la costa de $8,5 \%$. Es evidente que las inequidades sociales existen: ser pobre y haber nacido en la selva determina un modo de vida que, en la mayoría de los casos, perpetúa las diferencias acentuando los conflictos sociales.

Entonces si unimos ambas variables, acceso a la educación y maternidad no deseada, podemos observar que, en aquellas zonas -en especial la rural- donde casi se llega al 50\% de la población adolescente que no puede acceder a la escuela, es también el rango más vulnerable cuando se trata de embarazos con madres adolescentes.

Ciertamente, podría decir algún lector, muchos de los embarazos que ocurren en adolescentes son debidos a abuso sexual, así es, pero, la gran mayoría se da porque las jovencitas no están siendo formadas en una escuela, no cuentan con mayores expectativas para un mejor estilo de vida, para vislumbrar una manera de intentar, al menos, salir de ese circuito terrible de la pobreza que afecta a una familia, como mencionábamos al inicio, durante siete generaciones consecutivas. 
Por tanto, la educación es también un factor fundamental para acelerar la transición demográfica a tasas de natalidad y mortalidad más bajas en los países.

Sobretodo, cuando sabemos que todavía en algunos países muchas mujeres fallecen como consecuencia de las complicaciones sobrevenidas durante el embarazo o el parto. La educación puede evitar estas muertes, al ayudar a que las mujeres reconozcan los síntomas de peligro y busquen asistencia médica.

Según cifras de la UNICEF, si todas las mujeres hubieran cursado al menos la enseñanza primaria, la mortalidad materna se reduciría en dos tercios, con lo que cada año 189.000 mujeres salvarían la vida.

Sin embargo, la educación no solo ayuda a las personas a escapar de la pobreza mediante el desarrollo de las competencias que necesitan para mejorar sus medios de vida, también genera beneficios de productividad que potencian considerablemente el crecimiento económico. Así, la diferencia de los niveles de educación explica alrededor de la mitad de la diferencia en relación con el crecimiento.

En Guatemala, por ejemplo, los adultos apenas tenían 3,6 años de escolarización, en promedio, en 2005. Según cálculos realizados, si Guatemala hubiera igualado el promedio regional de avance educativo, podría haber más que duplicado su tasa de crecimiento medio anual entre 2005 y 2010.

Volviendo a nuestro país, el Perú, todos recordamos haber visto por televisión o escuchado en las noticias acerca de incendios forestales; son muy frecuentes; no me refiero a los que ocurren cuando se dan altísimas temperaturas, no, existe una costumbre pre inca que se denomina "rasa y quema", supongo que el rasa, viene del verbo arrasar, dejar sin nada un terreno...
La costumbre se inicia en la sierra, una familia decidía construir una casita en determinadolugar y habían árboles o arbustos crecidos, entonces, quemaba todo lo que había de vegetación para dejar el terreno liso para sembrar. En el ande, de acuerdo a las características del lugar, puede volverse a utilizar la tierra al año.

Sin embargo, en nuestro país, hay mucha movilidad de personas y muchos de la sierra se trasladan a la selva o ceja de selva y llevan consigo esta tradición ancestral, pero, el suelo y las condiciones climáticas son muy diferentes, así, la posibilidad de utilizar la tierra nuevamente no se da en un año como en la sierra, sino en un mínimo de tres años.

Entonces, es el caso de muchas personas que al no ver que, transcurrido un año, se puede volver a utilizar la tierra que fue quemada y de cuyas cenizas se hace abono, entonces, construyen su casa en otro lugar, y en otro..., y en otro..., repitiendo el proceso una y otra vez, con las terribles consecuencias para el medio ambiente que todos conocemos.

Sin embargo, ipor qué las conocemos? pues, porque este conocimiento se nos da a través de la educación que tenemos la suerte de recibir, a las personas que realizan estas quemas constantes de terrenos (que muchas veces desencadenan en incendios forestales de envergadura), probablemente, nadie les haya enseñado acerca del terrible daño ecológico que están generando..., eso también lo da la educación, nos reconcilia con nuestra gran casa que es la tierra, nos enseña a quererla y respetarla como a una madre amorosa que nos cuida y cobija.

Este año la UNIFÉ celebra los 50 años de su Facultad de Ciencias de la Educación, medio siglo formando mujeres educadoras, 50 años de vida fructífera en una sociedad compleja como es la peruana, 50 años transmitiendo a diario la luz del conocimiento, los ideales y valores 
que ayuden a convivir en paz y en armonía y, sobretodo, formando educadoras de calidad, ellas llevarán esa calidad a una serie de generaciones a su cuidado.

Y junto, con la UNIFÉ, no dejemos nunca de defender la educación de todos, en especial de las mujeres, que constituyen el segmento más proclive a ser olvidado en las zonas más pobres, donde el analfabetismo tiene rostro de mujer.

Si educamos a una mujer, lo hacemos con su familia, si lo hacemos con la familia, lo hacemos con la sociedad, si formamos educadoras convencidas de la trascendencia de su misión, educaremos a través de ellas a niños, jóvenes y adultos con miras a un Perú mejor.

\section{REFERENCIAS}

Basic Education Coalition (2004). Teach a Child, Transform a Nation (Transforma una nación dando educación a un niño). Washington y Banco Mundial 2002: Indicadores de desarrollo mundial. Washington. Citado en: Save the Children. 2005. El Estado Mundial de las Madres. Madrid.

Claramunt, M. C. (2000). Abuso sexual en mujeres adolescentes. http://www.paho.org/spanish/hdp/hdw/gph9.pdf

CEPAL, UNICEF (2007). Desafios. Boletín de la infancia y adolescencia sobre los objetivos de desarrollo del Milenio. Número 4, enero de 2007. Santiago de Chile.

Khan K. S.; Wojdyla D.; Say L.; Gulmezoglu A. M.; Van Look PFA. WHO analysis of causes of maternal death: a systematic review. Lancet 2006. Citado en: Chaparro, C. La anemia entre adolescentes y mujeres adultas jóvenes en América Latina y Empoderamiento de mujeres adolescentes.

Núñez, C. (2006). Educar para transformar, transformar para educar. Ed. Caminos.

OPS. S. F. Programa Mujer, Salud y Desarrollo. Equidad de género en salud. Disponible en: http://www.paho.org/spanish/ hdp/hdw/GEHPaperSpanish.pdf
Organización Mundial de la Salud/Centro Colaborador en Salud de Adolescentes, Universidad de Minnesota/ Organización Panamericana de la Salud (2000). A Portrait of Adolescent Health in the Caribbean. En: Schutt-Aine, J. y Maddaleno, M. 2003. Salud sexual y desarrollo de adolescentes y jóvenes en las Américas: Implicaciones en programas y políticas. Washington, D. C.: OPS.

UNICEF (1993), Sección de Educación-División de Programas Estrategias para Promover la Educación de las Niñas. Políticas y Programas que funcionan. Nueva York, Estados Unidos.

http://www.unicef.es/actualidad-documentacion/ noticias/escuelas-para-\%C3\%Alfrica-21-millones-deni\%C3\%Blos-escolarizados

http://www.unicef.es/sites/www.unicef.es/files/ crisismundial_Infancia.pdf

http://www.unwomen.org/es/news/stories/201l/4/ empowering-women-to-change-the-world-whatuniversities-and-the-un-can-do/\#sthash.76MfZXxh. dpuf 